\title{
EIGENVALUE PROBLEMS WITH WEIGHT AND VARIABLE EXPONENT FOR THE LAPLACE OPERATOR
}

\author{
MIHAI MIHĂILESCU ${ }^{*}, \dagger, \S$ and VICENŢIU RĂDULESCU*,‡,ף \\ *Department of Mathematics, University of Craiova \\ 200585 Craiova, Romania \\ ${ }^{\dagger}$ Department of Mathematics, Central European University \\ 1051 Budapest, Hungary \\ $\ddagger$ Institute of Mathematics "Simion Stoilow" of the Romanian Academy \\ P. O. Box 1-764, 014700 Bucharest, Romania \\ \$mmihailes@yahoo.com \\ 『vicentiu.radulescu@imar.ro
}

Received 7 January 2010

Accepted 10 February 2010

\begin{abstract}
This paper deals with an eigenvalue problem for the Laplace operator on a bounded domain with smooth boundary in $\mathbb{R}^{N}(N \geq 3)$. We establish that there exist two positive constants $\lambda_{\star}$ and $\lambda^{\star}$ with $\lambda_{\star} \leq \lambda^{\star}$ such that any $\lambda \in\left(0, \lambda_{\star}\right)$ is not an eigenvalue of the problem while any $\lambda \in\left[\lambda^{\star}, \infty\right)$ is an eigenvalue of the problem.
\end{abstract}

Keywords: Laplace operator; eigenvalue problem; critical point; spectrum.

Mathematics Subject Classification 2010: 35D05, 35J60, 35J70, 58E05, 15A18, 35P05

\section{Introduction}

This paper is motivated by the following simple problem. Assume that $\Omega \subset \mathbb{R}^{N}$ is a bounded domain with smooth boundary. Then the nonlinear Dirichlet problem

$$
\begin{cases}\Delta u=u^{3} & \text { in } \Omega \\ u=0 & \text { on } \partial \Omega\end{cases}
$$

does not have any nontrivial solution, as can be observed by multiplication by $u$ and integration. A natural question is to see what happens if problem (1.1) is affected by a certain perturbation. Let us consider the problem

$$
\begin{cases}-\Delta u=\lambda|u| u-u^{3} & \text { in } \Omega \\ u=0 & \text { on } \partial \Omega\end{cases}
$$

『 Corresponding author. 
where $\lambda$ is a positive parameter. Then

$$
\begin{aligned}
\int_{\Omega}|\nabla u|^{2} d x & =\lambda \int_{\Omega}|u|^{3} d x-\int_{\Omega} u^{4} d x \\
& \leq \lambda\left(\int_{\Omega} u^{4} d x\right)^{1 / 2}\left(\int_{\Omega} u^{2} d x\right)^{1 / 2}-\int_{\Omega} u^{4} d x \\
& \leq \frac{\lambda}{\sqrt{\lambda_{1, \Omega}}}\left(\int_{\Omega} u^{4} d x\right)^{1 / 2}\left(\int_{\Omega}|\nabla u|^{2} d x\right)^{1 / 2}-\int_{\Omega} u^{4} d x
\end{aligned}
$$

where $\lambda_{1, \Omega}$ denotes the first eigenvalue of the Laplace operator in $H_{0}^{1}(\Omega)$. We recall that $\lambda_{1, \Omega}$ can be characterized from a variational point of view as the minimum of the Rayleigh quotient, that is,

$$
\lambda_{1, \Omega}=\inf _{u \in H_{0}^{1}(\Omega) \backslash\{0\}} \frac{\int_{\Omega}|\nabla u|^{2} d x}{\int_{\Omega} u^{2} d x} .
$$

Moreover, it is known that $\lambda_{1, \Omega}$ is simple, that is, all the associated eigenfunctions are merely multiples of each other (see, e.g., [12]). Furthermore, the corresponding eigenfunctions of $\lambda_{1, \Omega}$ never change sign in $\Omega$.

Since

$$
\int_{\Omega}|\nabla u|^{2} d x+\int_{\Omega} u^{4} d x \geq 2\left(\int_{\Omega} u^{4} d x\right)^{1 / 2}\left(\int_{\Omega}|\nabla u|^{2} d x\right)^{1 / 2},
$$

relation (1.3) shows that a necessary condition for the existence of nontrivial solutions to problem (1.2) is that $\lambda \geq 2 \sqrt{\lambda_{1, \Omega}}$. This means that problem (1.2) does not have any nontrivial solution provided that $\lambda>0$ is small enough. Similar arguments show that the same conclusion holds if the term $\lambda|u| u$ in (1.2) is replaced by $\lambda|u|^{s-1} u$, where $1<s<3$.

In the present paper we are interested to obtain existence and nonexistence results for related nonlinear elliptic equations in a more general setting. We consider a model involving nonlinearities with variable exponent. The abstract framework is based on the theory of Lebesgue-Sobolev spaces with variable exponent. We point out that materials requiring such more advanced theory have been studied experimentally since the middle of the last century. The first major discovery is due to Willis Winslow in 1949 and is related to the theory of electrorheological fluids. These fluids have the interesting property that their viscosity depends on the electric field in the fluid. Winslow noticed that in such fluids (for instance, lithium polymetachrylate) viscosity in an electrical field is inversely proportional to the strength of the field. The field induces string-like formations in the fluid, which are parallel to the field. They can raise the viscosity by as much as five orders of magnitude. This phenomenon is known as the Winslow effect. For a general account of the underlying physics we refer to Halsey [13] and for some technical applications we refer to Pfeiffer et al. [26]. Electrorheological fluids have been used in robotics and space technology. 


\section{The Main Result}

In this paper we are concerned with the study of the eigenvalue problem

$$
\begin{cases}-\Delta u+|u|^{q(x)-2} u=\lambda g(x)|u|^{r(x)-2} u, & \text { for } x \in \Omega \\ u=0, & \text { for } x \in \partial \Omega,\end{cases}
$$

where $\Omega \subset \mathbb{R}^{N}$ is a bounded domain with smooth boundary, $q, r: \bar{\Omega} \rightarrow(1, \infty)$ are continuous functions verifying $2<\min _{\bar{\Omega}} r \leq \max _{\bar{\Omega}} r<\min _{\bar{\Omega}} q<2^{*}$, and $g: \Omega \rightarrow[0, \infty)$ is a bounded function for which there exists an open subset $\omega_{0} \subset \Omega$ such that $g(x)>0$ for any $x \in \omega_{0}$. We have denoted

$$
2^{*}= \begin{cases}\frac{2 N}{N-2} & \text { if } N \geq 3 \\ +\infty & \text { if } N \in\{1,2\} .\end{cases}
$$

The study of eigenvalue problems involving the Laplace operator is strongly related to a basic result in the elementary theory of partial differential equations which asserts that the linear problem

$$
\begin{cases}-\Delta u=\lambda u, & \text { in } \Omega \\ u=0, & \text { on } \partial \Omega\end{cases}
$$

has an unbounded sequence of eigenvalues $0<\lambda_{1, \Omega}<\lambda_{2, \Omega} \leq \cdots \leq \lambda_{n, \Omega} \leq \cdots$. This celebrated result goes back to the Riesz-Fredholm theory of self-adjoint and compact operators on Hilbert spaces (see [2]).

In a different context, we point out the inequality of Faber [8] and Krahn [15] (see also [16]) which states that

$$
\lambda_{1, \Omega} \geq \lambda_{1, B_{1}}|\Omega|^{-2 / N},
$$

where $\lambda_{1, B_{1}}$ is the lowest eigenvalue of problem (2.2) with $\Omega=B_{1}$, a ball of unit volume in $\mathbb{R}^{N}$. In other words, inequality (2.3) asserts that among all the domains $\Omega$, with a given volume $|\Omega|$, the ball has the smallest lowest eigenvalue. Moreover, by (2.3) it is clear that for a domain $\Omega$ with the volume $|\Omega|$ small, the eigenvalue $\lambda_{1, \Omega}$ is large.

Going further, another type of eigenvalue problem involving the Laplace operator is given by the nonlinear model equation

$$
\begin{cases}-\Delta u=\lambda|u|^{p(x)-2} u, & \text { in } \Omega \\ u=0, & \text { on } \partial \Omega,\end{cases}
$$

where $\Omega \subset \mathbb{R}^{N}(N \geq 3)$ is still a bounded domain with smooth boundary, while $p: \bar{\Omega} \rightarrow\left(1,2^{*}\right)$ is a given continuous function. (Obviously, the case when $p$ is a constant function on $\bar{\Omega}$ is allowed but we avoid the case when $p \equiv 2$ since this case is the object of problem (2.2), discussed above.) For this problem the growth rate of the variable exponent $p(\cdot)$ will be essential in the description of the set of 
eigenvalues. First, assuming that $\min _{\bar{\Omega}} p>2$, it can be proved (by using a mountainpass argument) that any $\lambda>0$ is an eigenvalue of problem (2.4). Next, in the case when $\min _{\bar{\Omega}} p<2$ it can be proved (by using Ekeland's variational principle) that the problem has a continuous family of eigenvalues which lies in a neighborhood of the origin (see, e.g., [21] or [9] for some extensions). Finally, we point out that the above result can be completed in the particular case when $\max _{\bar{\Omega}} p<2$. More exactly, in this situation it can be proved that the energy functional associated with problem (2.4) has a nontrivial (global) minimum point for any positive $\lambda$ large enough. In other words, if $\max _{\bar{\Omega}} p<2$ then there exist two positive constants $\mu_{1}$ and $\mu_{2}$ such that any $\lambda \in\left(0, \mu_{1}\right) \cup\left(\mu_{2}, \infty\right)$ is an eigenvalue of problem (2.4).

In what concerns the eigenvalue problems involving quasilinear operators we remember, in the case of homogeneous elliptic operators, the contributions of Anane [1], de Thélin [28, 29], Lindqvist [17] and Filippucci-Pucci-Rădulescu [11], while in the case of nonhomogeneous elliptic operators we point out the recent advances of Fan-Zhang-Zhao [10], Mihăilescu-Rădulescu [21-24], MihăilescuPucci-Rădulescu [18, 19], and Fan [9]. We also refer to the monographs by Ciarlet $[3,4]$ for various important applications to mathematical physics and geometry.

Motivated by the above results on problems (2.2) and (2.4) we consider it important to emphasize in what follows the situation offered by problem (2.1).

Undoubtedly, a weak solution corresponding to problem (2.1) will be sought in the Sobolev space $H_{0}^{1}(\Omega)$. We say that $\lambda \in \mathbb{R}$ is an eigenvalue of problem (2.1) if there exists $u \in H_{0}^{1}(\Omega) \backslash\{0\}$ such that

$$
\int_{\Omega} \nabla u \nabla v d x+\int_{\Omega}|u|^{q(x)-2} u v d x-\lambda \int_{\Omega} g(x)|u|^{r(x)-2} u v d x=0,
$$

for all $v \in H_{0}^{1}(\Omega)$. We point out that if $\lambda$ is an eigenvalue of problem (2.1) then the corresponding eigenfunction $u \in H_{0}^{1}(\Omega) \backslash\{0\}$ is a weak solution of problem (2.1).

The main result is given by the following theorem.

Theorem 1. There exist two positive constants $\lambda_{\star}$ and $\lambda^{\star}$ with $\lambda_{\star} \leq \lambda^{\star}$ such that any $\lambda \in\left(0, \lambda_{\star}\right)$ is not an eigenvalue of problem (2.1) while any $\lambda \in\left[\lambda^{\star}, \infty\right)$ is an eigenvalue of problem (2.1).

Remark 1. Unfortunately, Theorem 1 does not describe entirely the set of eigenvalues corresponding to problem (2.1). Actually, by Theorem 1 it is not clear if either $\lambda_{\star}=\lambda^{\star}$ or $\lambda_{\star}<\lambda^{\star}$. In the last case a natural question arises regarding the existence of eigenvalues in the interval $\left(\lambda_{\star}, \lambda^{\star}\right)$.

\section{Notations and Auxiliary Results}

Throughout this paper we denote by $\|\cdot\|$ the norm of the Hilbert space $H_{0}^{1}(\Omega)$, i.e.

$$
\|u\|=\left(\int_{\Omega}|\nabla u|^{2} d x\right)^{1 / 2}
$$


and for any real $p>1$ we denote by $|\cdot|_{p}$ the norm on the Lebesgue space $L^{p}(\Omega)$, i.e.

$$
|u|_{p}=\left(\int_{\Omega}|u|^{p} d x\right)^{1 / p} \quad \text { if } p \in(1, \infty),
$$

while for $p=\infty$ the norm on the Lebesgue space $L^{\infty}(\Omega)$ is defined by the following relation:

$$
|u|_{\infty}=\inf \{C>0 ;|u(x)| \leq C, \text { a.e. } x \in \Omega\} .
$$

Next, we highlight some basic results on the theory of Lebesgue-Sobolev spaces with variable exponent. For more details we refer to the book by Musielak [25] and the papers by Edmunds et al. [5-7], Kovacik and Rákosník [14], Mihăilescu and Rădulescu [20].

Set

$$
C_{+}(\bar{\Omega})=\{h ; h \in C(\bar{\Omega}), h(x)>1 \text { for all } x \in \bar{\Omega}\} .
$$

For any $h \in C_{+}(\bar{\Omega})$, we define

$$
h^{+}=\sup _{x \in \Omega} h(x) \quad \text { and } \quad h^{-}=\inf _{x \in \Omega} h(x) .
$$

For any $p \in C_{+}(\bar{\Omega})$, we define the variable exponent Lebesgue space $L^{p(x)}(\Omega)$

$$
=\left\{u ; u \text { is a measurable real-valued function such that } \int_{\Omega}|u(x)|^{p(x)} d x<\infty\right\} .
$$

We define on this space the Luxemburg norm by

$$
|u|_{p(x)}=\inf \left\{\mu>0 ; \int_{\Omega}\left|\frac{u(x)}{\mu}\right|^{p(x)} d x \leq 1\right\} .
$$

Let $L^{p^{\prime}(x)}(\Omega)$ denote the conjugate space of $L^{p(x)}(\Omega)$, where $1 / p(x)+1 / p^{\prime}(x)=1$. For any $u \in L^{p(x)}(\Omega)$ and $v \in L^{p^{\prime}(x)}(\Omega)$ the Hölder type inequality

$$
\left|\int_{\Omega} u v d x\right| \leq\left(\frac{1}{p^{-}}+\frac{1}{p^{\prime-}}\right)|u|_{p(x)}|v|_{p^{\prime}(x)}
$$

holds true.

An important role in manipulating the generalized Lebesgue-Sobolev spaces is played by the modular of the $L^{p(x)}(\Omega)$ space, which is the mapping $\rho_{p(x)}: L^{p(x)}(\Omega) \rightarrow$ $\mathbb{R}$ defined by

$$
\rho_{p(x)}(u)=\int_{\Omega}|u|^{p(x)} d x .
$$

If $\left(u_{n}\right), u \in L^{p(x)}(\Omega)$ then the following relations hold true

$$
|u|_{p(x)}>1 \Rightarrow|u|_{p(x)}^{p^{-}} \leq \rho_{p(x)}(u) \leq|u|_{p(x)}^{p^{+}}
$$




$$
\begin{gathered}
|u|_{p(x)}<1 \Rightarrow|u|_{p(x)}^{p^{+}} \leq \rho_{p(x)}(u) \leq|u|_{p(x)}^{p^{-}} \\
\left|u_{n}-u\right|_{p(x)} \rightarrow 0 \Leftrightarrow \rho_{p(x)}\left(u_{n}-u\right) \rightarrow 0 .
\end{gathered}
$$

Finally, we point out that if $s \in C_{+}(\bar{\Omega})$ and $s(x)<2^{*}$ for all $x \in \bar{\Omega}$ then the embedding $H_{0}^{1}(\Omega) \hookrightarrow L^{s(x)}(\Omega)$ is compact.

\section{Proof of the Main Result}

First, we will point out that there exists $\lambda_{\star}>0$ such that any $\lambda \in\left(0, \lambda_{\star}\right)$ is not an eigenvalue of problem (2.1). Indeed, let us define

$$
\lambda_{\star}=\inf _{u \in H_{0}^{1}(\Omega) \backslash\{0\}} \frac{\int_{\Omega}|\nabla u|^{2} d x+\int_{\Omega}|u|^{q(x)} d x}{\int_{\Omega} g(x)|u|^{r(x)} d x} .
$$

We show that $\lambda_{\star}>0$. In order to do that we point out first that by relation (1.4) there exists $\lambda_{1, \Omega}>0$ such that

$$
\int_{\Omega}|\nabla u|^{2} d x \geq \lambda_{1, \Omega} \int_{\Omega} u^{2} d x
$$

for any $u \in H_{0}^{1}(\Omega)$. On the other hand, we notice that using the fact that $2<$ $r(x)<q(x)$, for any $x \in \Omega$, we find

$$
u^{2}(x)+|u(x)|^{q} \geq|u(x)|^{r},
$$

for any $x \in \Omega$ and any $u \in H_{0}^{1}(\Omega)$. Integrating the above inequality over $\Omega$ we obtain

$$
\int_{\Omega} u^{2} d x+\int_{\Omega}|u|^{q(x)} d x \geq \int_{\Omega}|u|^{r(x)} d x \geq \frac{1}{|g|_{\infty}} \int_{\Omega} g(x)|u|^{r(x)} d x,
$$

for any $u \in H_{0}^{1}(\Omega)$. Combining relations (4.2) and (4.3) we get

$$
\int_{\Omega}|\nabla u|^{2} d x+\int_{\Omega}|u|^{q(x)} d x \geq \frac{1}{|g|_{\infty}} \min \left\{1, \lambda_{1, \Omega}\right\} \int_{\Omega} g(x)|u|^{r(x)} d x,
$$

for any $u \in H_{0}^{1}(\Omega)$. The last inequality shows that

$$
\lambda_{\star}>\frac{1}{|g|_{\infty}} \min \left\{1, \lambda_{1, \Omega}\right\}>0 .
$$

Let us now define, $J_{1}, I_{1}, J_{0}, I_{0}: H_{0}^{1}(\Omega) \rightarrow \mathbb{R}$ by

$$
J_{1}(u)=\frac{1}{2} \int_{\Omega}|\nabla u|^{2} d x+\int_{\Omega} \frac{1}{q(x)}|u|^{q(x)} d x, \quad I_{1}(u)=\int_{\Omega} \frac{1}{r(x)} g(x)|u|^{r(x)} d x,
$$

and

$$
J_{0}(u)=\int_{\Omega}|\nabla u|^{2} d x+\int_{\Omega}|u|^{q(x)} d x, \quad I_{0}(u)=\int_{\Omega} g(x)|u|^{r(x)} d x .
$$


Standard arguments imply that $J_{1}, I_{1} \in C^{1}\left(H_{0}^{1}(\Omega), \mathbb{R}\right)$ with

$$
\left\langle J_{1}^{\prime}(u), v\right\rangle=\int_{\Omega} \nabla u \nabla v d x+\int_{\Omega}|u|^{q(x)-2} u v d x,
$$

and

$$
\left\langle I_{1}^{\prime}(u), v\right\rangle=\int_{\Omega} g(x)|u|^{r(x)-2} u v d x,
$$

for any $u, v \in H_{0}^{1}(\Omega)$.

Lemma 1. Assume $\lambda_{\star}$ is defined by relation (4.1). Then, any $\lambda \in\left(0, \lambda_{\star}\right)$ is not an eigenvalue of problem (2.1).

Proof. Indeed, assuming by contradiction that there exists $\lambda \in\left(0, \lambda_{\star}\right)$ an eigenvalue of problem (2.1), it follows that we can find $w_{\lambda} \in H_{0}^{1}(\Omega) \backslash\{0\}$ such that

$$
\left\langle J_{1}^{\prime}\left(w_{\lambda}\right), v\right\rangle=\lambda\left\langle I_{1}^{\prime}\left(w_{\lambda}\right), v\right\rangle
$$

for any $v \in H_{0}^{1}(\Omega)$. Letting $v=w_{\lambda}$, we deduce

$$
\left\langle J_{1}^{\prime}\left(w_{\lambda}\right), w_{\lambda}\right\rangle=\lambda\left\langle I_{1}^{\prime}\left(w_{\lambda}\right), w_{\lambda}\right\rangle,
$$

or

$$
J_{0}\left(w_{\lambda}\right)=\lambda I_{0}\left(w_{\lambda}\right) .
$$

Since $w_{\lambda} \neq 0$ we deduce that $J_{0}\left(w_{\lambda}\right)>0$ and thus, $I_{0}\left(w_{\lambda}\right)>0$. Combining that fact with the ideas that $\lambda \in\left(0, \lambda_{\star}\right)$ and $\lambda_{\star}=\inf _{u \in H_{0}^{1}(\Omega) \backslash\{0\}} \frac{J_{0}(u)}{I_{0}(u)}$, we infer

$$
J_{0}\left(w_{\lambda}\right) \geq \lambda_{\star} I_{0}\left(w_{\lambda}\right)>\lambda I_{0}\left(w_{\lambda}\right)=J_{0}\left(w_{\lambda}\right)
$$

and that is a contradiction. The proof of Lemma 1 is complete.

Next, for any $\lambda>0$ we define the functional associated with problem (2.1), $T_{\lambda}: H_{0}^{1}(\Omega) \rightarrow \mathbb{R}$ by

$$
T_{\lambda}(u)=J_{1}(u)-\lambda \cdot I_{1}(u), \quad \forall u \in H_{0}^{1}(\Omega) .
$$

It is clear that $\lambda$ is an eigenvalue for problem (2.1) if and only if there exists $u_{\lambda} \in H_{0}^{1}(\Omega) \backslash\{0\}$, a critical point of the functional $T_{\lambda}$.

Define

$$
\lambda^{\star}=\inf _{u \in H_{0}^{1}(\Omega) \backslash\{0\}} \frac{J_{1}(u)}{I_{1}(u)} .
$$

Using the same method as in the proof of the fact that $\lambda_{\star}>0$, we can deduce that

$$
\frac{J_{1}(u)}{I_{1}(u)} \geq \min \left\{\frac{\lambda_{1, \Omega}}{2}, \frac{1}{q^{+}}\right\} \cdot \frac{r^{-}}{|g|_{\infty}}, \quad \forall u \in H_{0}^{1}(\Omega),
$$

and thus,

$$
\lambda^{\star} \geq \min \left\{\frac{\lambda_{1, \Omega}}{2}, \frac{1}{q^{+}}\right\} \cdot \frac{r^{-}}{|g|_{\infty}}>0 .
$$


Lemma 2. For any $\lambda \in(0, \infty)$ we have

$$
\lim _{\|u\| \rightarrow \infty} T_{\lambda}(u)=\infty
$$

Proof. It is clear that by relation (4.2) and the fact that $2<r^{-} \leq r^{+}<q^{-}$ we have

$$
\begin{aligned}
T_{\lambda}(u) & =\frac{1}{2} \int_{\Omega}|\nabla u|^{2} d x+\int_{\Omega} \frac{1}{q(x)}|u|^{q(x)} d x-\lambda \int_{\Omega} \frac{g(x)}{r(x)}|u|^{r(x)} d x \\
& \geq \frac{1}{2}\|u\|^{2}+C_{1} \int_{\Omega}|u|^{q(x)} d x-C_{2} \int_{\Omega}|u|^{r(x)} d x \\
& \geq \frac{1}{4}\|u\|^{2}+\frac{\lambda_{1}, \Omega}{4} \int_{\Omega} u^{2} d x+C_{1} \int_{\Omega}|u|^{q(x)} d x-C_{2} \int_{\Omega}\left(|u|^{r^{+}}+|u|^{r^{-}}\right) d x \\
& \geq \frac{1}{4}\|u\|^{2}+C_{3} \int_{\Omega}|u|^{s} d x-C_{2} \int_{\Omega}\left(|u|^{r^{+}}+|u|^{r^{-}}\right) d x
\end{aligned}
$$

for any $u \in H_{0}^{1}(\Omega)$, where $s \in\left(2, q^{-}\right)$is a fixed real number, while $C_{1}, C_{2}$ and $C_{3}$ are three positive constants. Moreover, since $s>r^{ \pm}$, it follows by the Lebesgue embeddings that there exist two positive constants $C_{ \pm}$such that

$$
|u|_{r^{ \pm}}^{r^{ \pm}} \leq C_{ \pm}|u|_{s}^{r^{ \pm}}, \quad \forall u \in H_{0}^{1}(\Omega)
$$

Combining the last two relations, we infer that for any $u \in H_{0}^{1}(\Omega)$ we have

$$
T_{\lambda}(u) \geq \frac{1}{4}\|u\|^{2}+\left(\frac{C_{3}}{2}|u|_{s}^{s}-C_{4}|u|_{s}^{r^{-}}\right)+\left(\frac{C_{3}}{2}|u|_{s}^{s}-C_{4}|u|_{s}^{r^{+}}\right),
$$

where $C_{4}$ and $C_{5}$ are two positive constants.

Next, we show that for any $u \in H_{0}^{1}(\Omega)$ there exist two positive constants $M_{i}=$ $M_{i}\left(r, q, C_{3}, C_{4}\right)$, with $i \in\{1,2\}$, and such that

$$
\frac{C_{3}}{2}|u|_{s}^{s}-C_{4}|u|_{s}^{r^{-}} \geq-M_{1}
$$

and

$$
\frac{C_{3}}{2}|u|_{s}^{s}-C_{4}|u|_{s}^{r^{+}} \geq-M_{2}
$$

In order to prove that, we point out that the functional $\Phi:(0, \infty) \rightarrow \infty$ defined by

$$
\Phi(t)=\alpha \cdot t^{a}-\beta \cdot t^{b}
$$

where $\alpha, \beta, a, b$ are positive constants with $a<b$, achieves its positive global maximum

$$
\Phi\left(t_{0}\right)=\frac{b-a}{a} \cdot\left(\frac{a}{b}\right)^{a /(b-a)} \cdot \alpha^{b /(b-a)} \cdot \beta^{a /(a-b)}>0
$$


where $t_{0}=((\alpha \cdot a) /(\beta \cdot b))^{1 /(b-a)}>0$. Thus, we deduce that

$$
\alpha \cdot t^{a}-\beta \cdot t^{b} \leq C(a, b) \cdot \alpha^{b /(b-a)} \cdot \beta^{a /(a-b)}, \quad \forall t>0,
$$

where $C(a, b)=(b-a) \cdot\left(\frac{a^{a}}{b^{b}}\right)^{1 /(b-a)}$.

Relations (4.8)-(4.10) show that the conclusion of Lemma 2 holds true.

Lemma 3. Any $\lambda \in\left(\lambda^{\star}, \infty\right)$ is an eigenvalue of problem (2.1).

Proof. We fix $\lambda \in\left(\lambda^{\star}, \infty\right)$. By Lemma 2 we deduce that $\lim _{\|u\| \rightarrow \infty} T_{\lambda}(u)=\infty$, i.e. $T_{\lambda}$ is coercive. On the other hand, it is clear that the functional $T_{\lambda}$ is weakly lower semi-continuous. These two facts enable us to apply [27, Theorem 1.2] in order to prove that there exists $u_{\lambda} \in H_{0}^{1}(\Omega)$, a global minimum point of $T_{\lambda}$.

Next, we show that $u_{\lambda}$ is not trivial. Indeed, since $\lambda^{\star}=\inf _{u \in H_{0}^{1}(\Omega) \backslash\{0\}} \frac{J_{1}(u)}{I_{1}(u)}$ and $\lambda>\lambda^{\star}$, it follows that there exists $v_{\lambda} \in H_{0}^{1}(\Omega)$ such that

$$
J_{1}\left(v_{\lambda}\right)<\lambda I_{1}\left(v_{\lambda}\right)
$$

or

$$
T_{\lambda}\left(v_{\lambda}\right)<0
$$

Thus,

$$
\inf _{H_{0}^{1}(\Omega)} T_{\lambda}<0
$$

and we conclude that $u_{\lambda} \neq 0$.

Next, we show that $\lambda$ is an eigenvalue of problem (2.1). We notice that if $v \in$ $H_{0}^{1}(\Omega)$ then, since $u_{\lambda} \neq 0$, it follows that for each $\epsilon>0$ small enough we have $\left(u_{\lambda}+\epsilon v\right) \neq 0$. Thus, we deduce

$$
\left.\frac{d}{d \epsilon} T_{\lambda}\left(u_{\lambda}+\epsilon v\right)\right|_{\epsilon=0}=0
$$

or

$$
\left\langle J_{1}^{\prime}\left(u_{\lambda}\right), v\right\rangle-\lambda\left\langle I_{1}^{\prime}\left(u_{\lambda}\right), v\right\rangle=0, \quad \forall v \in H_{0}^{1}(\Omega) .
$$

Thus, $\lambda$ is an eigenvalue of problem (2.1). The proof of Lemma 3 is complete.

Finally, we concentrate our efforts to show that $\lambda^{\star}$, given by relation (4.6), is also an eigenvalue of problem (2.1). In order to do that we prove first the following auxiliary result:

\section{Lemma 4.}

$$
\lim _{\|u\| \rightarrow 0} \frac{J_{0}(u)}{I_{0}(u)}=\infty .
$$

Proof. Since $2<r^{-} \leq r^{+}<2^{*}$ it follows that the following continuous embeddings hold true

$$
H_{0}^{1}(\Omega) \subset L^{r^{ \pm}}(\Omega) .
$$


Consequently, there exists a positive constant $D$ such that

$$
\begin{aligned}
\int_{\Omega} g(x)|u|^{r(x)} d x & \leq|g|_{\infty} \int_{\Omega}|u|^{r(x)} d x \\
& \leq|g|_{\infty} \int_{\Omega}\left(|u|^{r^{-}}+|u|^{r^{+}}\right) d x \\
& \leq D \cdot\left(\|u\|^{r^{-}}+\|u\|^{r^{+}}\right), \quad \forall u \in H_{0}^{1}(\Omega) .
\end{aligned}
$$

Using the above inequality we obtain the estimates

$$
\frac{\int_{\Omega}|\nabla u|^{2} d x+\int_{\Omega}|u|^{q(x)} d x}{\int_{\Omega} g(x)|u|^{r(x)} d x} \geq \frac{\|u\|^{2}}{D \cdot\left(\|u\|^{r^{-}}+\|u\|^{r^{+}}\right)}, \quad \forall u \in H_{0}^{1}(\Omega) .
$$

Since $r^{ \pm}>2$ it follows that passing to the limit as $\|u\| \rightarrow 0$ in the above inequality, we deduce that Lemma 4 holds true.

Lemma 5. The real number $\lambda^{\star}$, given by relation (4.6), is an eigenvalue (2.1).

Proof. Let $\left(\lambda_{n}\right)$ be a sequence in $\mathbb{R}$ such that $\lambda_{n} \searrow \lambda^{\star}$ as $n \rightarrow \infty$. By Lemma 3 we deduce that for each $n$ there exists $u_{n} \in H_{0}^{1}(\Omega) \backslash\{0\}$ such that

$$
\left\langle J_{1}^{\prime}\left(u_{n}\right), v\right\rangle-\lambda_{n}\left\langle I_{1}^{\prime}\left(u_{n}\right), v\right\rangle=0, \quad \forall v \in H_{0}^{1}(\Omega) .
$$

Taking $v=u_{n}$ in the above equality, we find

$$
J_{0}\left(u_{n}\right)=\lambda_{n} I_{0}\left(u_{n}\right), \quad \forall n .
$$

The above equality and Lemma 2 imply that $\left(u_{n}\right)$ is a bounded sequence in $H_{0}^{1}(\Omega)$. Indeed, assuming by contradiction that $\left(u_{n}\right)$ is not bounded in $H_{0}^{1}(\Omega)$ it follows that passing, if necessary, to a subsequence, still denoted by $\left(u_{n}\right)$, we have $\left\|u_{n}\right\| \rightarrow \infty$. On the other hand, the fact that $\lambda_{n} \searrow \lambda^{\star}$ and relation (4.12) imply that for each $n$ large enough it holds true that

$$
J_{0}\left(u_{n}\right)=\lambda_{n} I_{0}\left(u_{n}\right) \leq\left(\lambda^{\star}+1\right) I_{0}\left(u_{n}\right) .
$$

Lemma 2 shows that the above inequality and the fact that $\left\|u_{n}\right\| \rightarrow \infty$ lead to a contradiction. Consequently, $\left(u_{n}\right)$ is bounded in $H_{0}^{1}(\Omega)$. We deduce the existence of $u \in H_{0}^{1}(\Omega)$ such that $u_{n}$ converges weakly to $u$ in $H_{1}$. Since $q(x), r(x) \in\left(2,2^{*}\right)$ the Rellich-Kondrachov compact embedding theorem shows that $u_{n}$ converges strongly to $u$ in $L^{q(x)}(\Omega)$ and in $L^{r(x)}(\Omega)$. Thus, passing to the limit as $n \rightarrow \infty$ in $(4.11)$ we get

$$
\left\langle J_{1}^{\prime}(u), v\right\rangle-\lambda^{\star}\left\langle I_{1}^{\prime}(u), v\right\rangle=0, \quad \forall v \in H_{0}^{1}(\Omega) .
$$

Thus, $\lambda$ is an eigenvalue of problem (2.1), provided that $u \neq 0$. 
On the other hand, we point out that taking $v=u$ in the above equality we find

$$
J_{0}(u)=\lambda^{\star} I_{0}(u) .
$$

We also point out that passing to the limit as $n \rightarrow \infty$ in (4.12), we obtain

$$
\lim _{n \rightarrow \infty} J_{0}\left(u_{n}\right)=\lambda^{\star} I_{0}(u) .
$$

Combining that last two equalities we infer that, actually, $u_{n}$ converges strongly to $u$ in $H_{0}^{1}(\Omega)$.

Now, we are ready to prove that $u \neq 0$. Assuming by contradiction that $u=0$ we deduce that $u_{n}$ converges strongly to $u$ in $H_{0}^{1}(\Omega)$. By relation (4.12) we deduce that for any $n$ the equality

$$
\frac{J_{0}\left(u_{n}\right)}{I_{0}\left(u_{n}\right)}=\lambda_{n}
$$

holds. Passing to the limit as $n \rightarrow \infty$ and taking into account the result of Lemma 4 and the fact that $\lambda_{n} \searrow \lambda^{\star}$, we obtain a contradiction. Consequently, $u \neq 0$, and, thus, $\lambda^{\star}$ is an eigenvalue of problem (2.1). The proof of Lemma 5 is complete.

Obviously, the numbers $\lambda_{\star}$ and $\lambda^{\star}$ defined by relations (4.1) and (4.6) verify $\lambda_{\star} \leq \lambda^{\star}$. Moreover, the estimates in Theorem 1 are valid since relations (4.5) and (4.7) hold true. The conclusion of Theorem 1 follows by Lemmas 1, 3 and 5 .

\section{Acknowledgments}

The authors have been fully supported by Grant CNCSIS 79/2007 "Degenerate and Singular Nonlinear Processes" (M. Mihăilescu) and Grant CNCSIS PCCE-55/2008 "Differential Systems in Nonlinear Analysis and Applications" (V. Rădulescu).

\section{References}

[1] A. Anane, Simplicité et isolation de la première valeur propre du $p$-Laplacien avec poids, C. R. Acad. Sci. Paris Sér. I Math. 305 (1987) 725-728.

[2] H. Brezis, Functional Analysis, Sobolev Spaces and Partial Differential Equations (Springer, New York, 2010).

[3] P. G. Ciarlet, The Finite Element Method for Elliptic Problems, Studies in Mathematics and its Applications, Vol. 4 (North-Holland Publishing Co., Amsterdam-New York-Oxford, 1978).

[4] P. G. Ciarlet, An Introduction to Differential Geometry with Applications to Elasticity (Springer, Dordrecht, 2005).

[5] D. E. Edmunds, J. Lang and A. Nekvinda, On $L^{p(x)}$ norms, Proc. Roy. Soc. London Ser. A 455 (1999) 219-225.

[6] D. E. Edmunds and J. Rákosník, Density of smooth functions in $W^{k, p(x)}(\Omega)$, Proc. Roy. Soc. London Ser. A 437 (1992) 229-236.

[7] D. E. Edmunds and J. Rákosník, Sobolev embedding with variable exponent, Studia Math. 143 (2000) 267-293. 
[8] C. Faber, Beweis das unter allen homogenen Membranen yon gleicher Fläche und gleicher Spannung die kreisförmige den tiefsten Grundton gibt, Sitzungsber. Bayer. Akad. der Wiss. Math. Phys. (1923), pp. 169-172.

[9] X. Fan, Remarks on eigenvalue problems involving the $p(x)$-Laplacian, J. Math. Anal. Appl. 352 (2009) 85-98.

[10] X. Fan, Q. Zhang and D. Zhao, Eigenvalues of $p(x)$-Laplacian Dirichlet problem, J. Math. Anal. Appl. 302 (2005) 306-317.

[11] R. Filippucci, P. Pucci and V. Rădulescu, Existence and non-existence results for quasilinear elliptic exterior problems with nonlinear boundary conditions, Comm. Partial Differential Equations 33 (2008) 706-717.

[12] D. Gilbarg and N. S. Trudinger, Elliptic Partial Differential Equations of Second Order (Springer, Berlin, 1998).

[13] T. C. Halsey, Electrorheological fluids, Science 258 (1992) 761-766.

[14] O. Kováčik and J. Rákosník, On spaces $L^{p(x)}$ and $W^{1, p(x)}$, Czechoslovak Math. J. 41 (1991) 592-618.

[15] E. Krahn, Über eine von Rayleigh formulierte Minimaleigenschaft des Kreises, Math. Ann. 94 (1925) 97-100.

[16] E. H. Lieb, On the lowest eigenvalue of the Laplacian for the intersection of two domains, Invent. Math. 74 (1983) 441-448.

[17] P. Lindqvist, On the equation $\operatorname{div}\left(|\nabla u|^{p-2} \nabla u\right)+\lambda|u|^{p-2} u=0$, Proc. Amer. Math. Soc. 109 (1990) 157-164.

[18] M. Mihăilescu, P. Pucci and V. Rădulescu, Nonhomogeneous boundary value problems in anisotropic Sobolev spaces, C. R. Acad. Sci. Paris, Sér. I 345 (2007) 561-566.

[19] M. Mihăilescu, P. Pucci and V. Rădulescu, Eigenvalue problems for anisotropic quasilinear elliptic equations with variable exponent, J. Math. Anal. Appl. 340 (2008) 687-698.

[20] M. Mihăilescu and V. Rădulescu, A multiplicity result for a nonlinear degenerate problem arising in the theory of electrorheological fluids, Proc. R. Soc. Lond. Ser. A Math. Phys. Eng. Sci. 462 (2006) 2625-2641.

[21] M. Mihăilescu and V. Rădulescu, On a nonhomogeneous quasilinear eigenvalue problem in Sobolev spaces with variable exponent, Proc. Amer. Math. Soc. 135 (2007) 2929-2937.

[22] M. Mihăilescu and V. Rădulescu, Eigenvalue problems associated to nonhomogeneous differential operators in Orlicz-Sobolev spaces, Anal. Appl. (Singap.) 6 (2008) 83-98.

[23] M. Mihăilescu and V. Rădulescu, Continuous spectrum for a class of nonhomogeneous differential operators, Manuscripta Math. 125 (2008) 157-167.

[24] M. Mihăilescu and V. Rădulescu, Spectrum consisting in an unbounded interval for a class of nonhomogeneous differential operators, Bull. London Math. Soc. 40 (2008) 972-984.

[25] J. Musielak, Orlicz Spaces and Modular Spaces, Lecture Notes in Mathematics, Vol. 1034 (Springer-Verlag, Berlin, 1983).

[26] C. Pfeiffer, C. Mavroidis, Y. Bar-Cohen and B. Dolgin, Electrorheological fluid based force feedback device, in Proc. SPIE Telemanipulator and Telepresence Technologies VI Conf. (Boston, MA), Vol. 3840 (SPIF, 1999), pp. 88-99.

[27] M. Struwe, Variational Methods: Applications to Nonlinear Partial Differential Equations and Hamiltonian Systems (Springer, Heidelberg, 1996).

[28] F. de Thélin, Quelques résultats d'existence et de non-existence pour une E.D.P. elliptique non linéaire, C. R. Acad. Sci. Paris Sér. I Math. 299 (1984) 911-914.

[29] F. de Thélin, Sur l'espace propre associé à la première valeur propre du pseudolaplacien, C. R. Acad. Sci. Paris Sér. I Math. 303 (1986) 355-358. 